\title{
Haemodynamically proven pulmonary hypertension in a patient with GATA2 deficiency-associated pulmonary alveolar proteinosis and fibrosis
}

\author{
To the Editor:
}

We read with great interest the article by BALLERIE et al. [1], which describes the occurrence of pulmonary alveolar proteinosis and fibrosis in a patient with GATA2 deficiency. Interestingly, our team recently managed a similar case, in which the interstitial lung disease eventually led to pulmonary hypertension $(\mathrm{PH})$.

A 41-year-old woman was referred to our tertiary care centre in 2014 for a suspected primary immunodeficiency. Her previous medical history included recurrent upper and lower respiratory tract infections, persisting cytopenias (including monocytopenia, and B-cell and natural killer cell lymphopenias), a disseminated Mycobacterium kansasii infection (in 2002) and a myelodysplastic syndrome (in 2008). She also displayed an interstitial lung disease that was first noted on a chest high-resolution computed tomography (HRCT) in 2002. A lung biopsy performed in 2007 showed histological lesions of pulmonary alveolar proteinosis and paraseptal fibrosis. Over the years, the pulmonary disease slowly progressed to involve the whole lung parenchyma. At the time of referral, chest HRCT displayed diffuse interlobular septal thickening, reticulations and ground-glass opacities of both lungs (figure 1a). The overall picture was compatible with the diagnosis of GATA2 deficiency, which was confirmed by molecular biology in October 2014.

At the time of GATA2 deficiency diagnosis, the patient complained of a slowly progressive worsening of her dyspnoea and was staged in New York Heart Association functional class IIa. Physical examination showed no sign of right heart failure (including lower limb oedema). The 6-min walk test confirmed limited exercise capacity with a walked distance of $350 \mathrm{~m}$ (53\% of the predicted value) without desaturation. Chest HRCT was controlled and showed stable interstitial anomalies, and helical computed tomography of the chest excluded a pulmonary embolism. Pulmonary function tests displayed a restrictive syndrome (with a total lung capacity of $76 \%$ and a forced vital capacity of $55 \%$ of their predicted values), a mild hypoxaemia (oxygen tension $81 \mathrm{mmHg}$ and carbon dioxide tension $29 \mathrm{mmHg}$ in room air) and a marked alteration in diffusing capacity of the lung for carbon monoxide (36\% of the predicted value). Serum levels of $\mathrm{N}$-terminal pro-brain natriuretic peptide were slightly elevated at $416 \mathrm{pg} \cdot \mathrm{mL}^{-1}$ (normally $<300 \mathrm{pg} \cdot \mathrm{mL}^{-1}$ ). A transthoracic echocardiography was thus performed and showed signs suggestive of $\mathrm{PH}$ (estimated systolic pulmonary arterial pressure (PAP) $66 \mathrm{mmHg}$ ) and right ventricle dilation (right-to-left ventricle diameter ratio 1.15), with no sign of diastolic or systolic left heart dysfunction.

A right heart catheterisation performed in October 2015 revealed a moderate pre-capillary $\mathrm{PH}$ (systolic/ diastolic/mean PAP 48/17/32 mmHg, pulmonary vascular resistance 6.0 Wood units, pulmonary arterial wedge pressure $6 \mathrm{mmHg}$ and normal portal pressure gradient) with a preserved cardiac function (cardiac output $4.30 \mathrm{~L} \cdot \mathrm{min}^{-1}$ and cardiac index $3.33 \mathrm{~L} \cdot \mathrm{min}^{-1} \cdot \mathrm{m}^{-2}$ ). The patient was considered to have $\mathrm{PH}$ due to lung disease (World Health Organization group 3 [2]). As such, pulmonary arterial hypertension-specific therapies were not introduced. Clinical, functional and echographic parameters remained stable after 12 months.

GATA2 deficiency is a novel entity characterised by heterozygous mutations in the GATA2 transcription factor gene [3]. It is associated with a wide spectrum of clinical features, including atypical and/or

@ERSpublications

Pulmonary hypertension can occur in GATA2 deficiency-associated pulmonary alveolar proteinosis and fibrosis http://ow.ly/aHv430aAZct

Cite this article as: Sanges S, Prévotat A, Fertin M, et al. Haemodynamically proven pulmonary hypertension in a patient with GATA2 deficiency-associated pulmonary alveolar proteinosis and fibrosis. Eur Respir J 2017; 49: 1700178 [https://doi.org/10.1183/13993003.00178-2017]. 

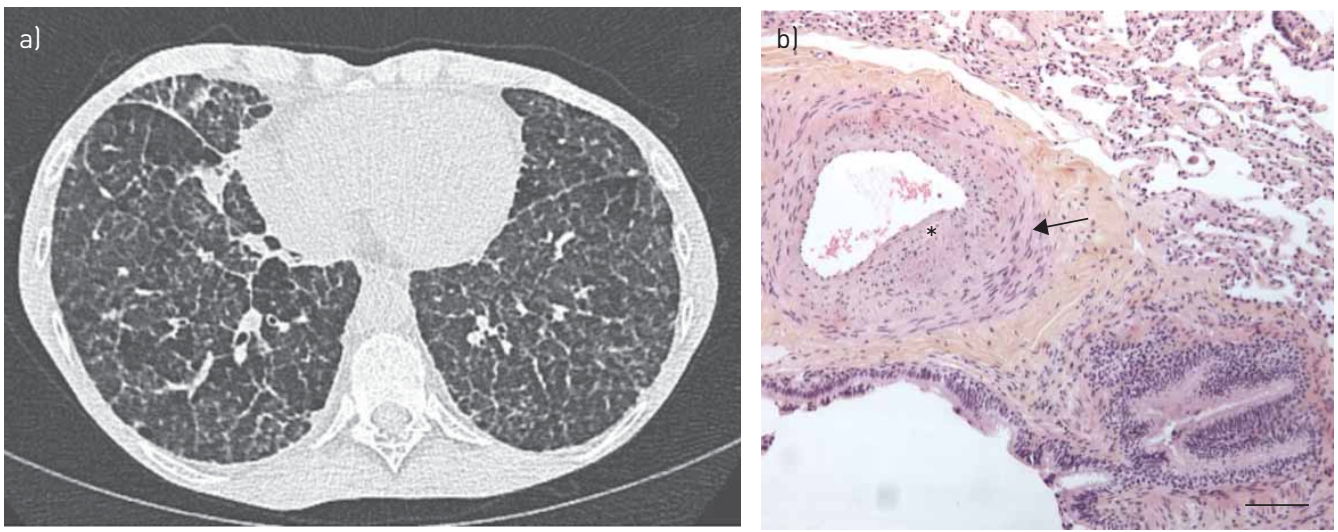

FIGURE 1 a) Chest high-resolution computed tomography image of our patient, showing diffuse interlobular septal thickening, reticulations and ground-glass opacities in both lungs. b) Lung biopsy of our patient (haematoxylin and eosin staining) showing a pulmonary artery adjacent to a respiratory bronchiole in a bronchovascular bundle. The arterial wall is slightly thickened by a medial smooth muscle hyperplasia (arrow) and an intimal fibrosis (asterisk), without vasculitis. Scale bar=120 $\mu \mathrm{m}$.

recurrent infections (notably with herpesviruses, papillomaviruses and nontuberculous mycobacteria), cytopenias (especially monocytopenia), haematological malignancies (myelodysplasia, acute myeloblastic leukaemia and chronic myelomonocytic leukaemia), vascular complications (deep vein thrombosis and lymphoedema) and interstitial lung disease (mainly pulmonary alveolar proteinosis) [4]. Previous publications listed $\mathrm{PH}$ among the possible complications of the disease [5-7], occurring in as many as $9 \%$ of cases [6]. However, the detailed characteristics of these patients (especially in terms of haemodynamic profile) were not provided and the precise mechanisms underlying $\mathrm{PH}$ were never investigated.

At the end of a comprehensive evaluation, our patient was diagnosed with $\mathrm{PH}$ due to a long-lasting pulmonary alveolar proteinosis and fibrosis. As GATA2 plays a crucial role in regulating several endothelial cell functions [8], another putative mechanism for $\mathrm{PH}$ in this case could also be a specific GATA2 deficiency-induced vasculopathy. In a previous work, SvoBodova et al. [9] described pulmonary vascular anomalies (thickened arterial wall, destruction of the elastic layer and thrombosis showing organising vasculitis) in a patient with GATA2 deficiency-associated interstitial lung disease. Interestingly, some similar histological findings were observed in the 2007 lung biopsy of our patient (figure 1b) and an echocardiography performed around that time already showed early signs compatible with $\mathrm{PH}$ (estimated systolic PAP $43 \mathrm{mmHg}$ ). Whether these anomalies are related to $\mathrm{PH}$, either as a cause or a consequence, remains uncertain.

Therapeutic options in GATA2 deficiency are currently limited. A recent work suggested a possible efficacy of allogeneic haematopoietic stem cell transplantation on cytopenias and myelodysplastic syndrome in a series of six patients [5]. Interestingly, two of them were reported to have $\mathrm{PH}$ and pulmonary alveolar proteinosis, and their respiratory condition was described as improved after transplantation [6].

To conclude, in addition to the association of pulmonary alveolar proteinosis and fibrosis described by BALLERIE et al. [1], physicians should be aware of the possible occurrence of PH in patients with GATA2 deficiency.

Sébastien Sanges ${ }^{1,2,3,4}$, Anne Prévotat $^{5}$, Marie Fertin ${ }^{6}$, Louis Terriou ${ }^{1,2,3,4}$, Guillaume Lefèvre ${ }^{1,2,3,4,7}$, Bruno Quesnel ${ }^{8}$, Pierre-Yves Hatron ${ }^{1,3,4}$, Éric Hachulla ${ }^{1,2,3,4}$, Marie-Christine Copin' and David Launay ${ }^{1,2,3,4}$

${ }^{1}$ Univ. Lille, INSERM U995 - LIRIC - Lille Inflammation Research International Center, Lille, France. ${ }^{2}$ INSERM, U995, Lille, France. ${ }^{3} \mathrm{CHU}$ Lille, Département de Médecine Interne et Immunologie Clinique, Lille, France. ${ }^{4}$ Centre National de Référence Maladies Systémiques et Auto-immunes Rares (Sclérodermie Systémique), Lille, France. ${ }^{5} \mathrm{CHU}$ Lille, Service de Pneumologie, Lille, France. ${ }^{6} \mathrm{CHU}$ Lille, Service de Cardiologie, Lille, France. ${ }^{7} \mathrm{CHU}$ Lille, Institut d'Immunologie, Lille, France. ${ }^{8} \mathrm{CHU}$ Lille, Service des Maladies du Sang, Lille, France. ${ }^{9} \mathrm{CHU}$ Lille, Institut de Pathologie, Lille, France.

Correspondence: David Launay, Service de Médecine Interne et d'Immunologie Clinique, Hôpital Claude-Huriez, CHRU Lille, Rue Michel Polonovski, 59037 Lille Cedex, France. E-mail: david.launay@univ-lille2.fr

Received: Jan 242017 | Accepted after revision: Feb 022017

Conflict of interest: None declared. 


\section{References}

1 Ballerie A, Nimubona S, Meunier C, et al. Association of pulmonary alveolar proteinosis and fibrosis: patient with GATA2 deficiency. Eur Respir J 2016; 48: 1510-1514.

2 Simonneau G, Gatzoulis MA, Adatia I, et al. Updated clinical classification of pulmonary hypertension. J Am Coll Cardiol 2013; 62: D34-D41.

3 Hsu AP, Sampaio EP, Khan J, et al. Mutations in GATA2 are associated with the autosomal dominant and sporadic monocytopenia and mycobacterial infection (MonoMAC) syndrome. Blood 2011; 118: 2653-2655.

4 Hsu AP, McReynolds LJ, Holland SM. GATA2 deficiency. Curr Opin Allergy Clin Immunol 2015; 15: 104-109.

5 Cuellar-Rodriguez J, Gea-Banacloche J, Freeman AF, et al. Successful allogeneic hematopoietic stem cell transplantation for GATA2 deficiency. Blood 2011; 118: 3715-3720.

6 Spinner MA, Sanchez LA, Hsu AP, et al. GATA2 deficiency: a protean disorder of hematopoiesis, lymphatics, and immunity. Blood 2014; 123: 809-821.

7 Griese M, Zarbock R, Costabel U, et al. GATA2 deficiency in children and adults with severe pulmonary alveolar proteinosis and hematologic disorders. BMC Pulm Med 2015; 15: 87.

8 Hartmann D, Fiedler J, Sonnenschein K, et al. MicroRNA-based therapy of Gata2-deficient vascular disease. Circulation 2016; 134: 1973-1990.

9 Svobodova T, Mejstrikova E, Salzer U, et al. Diffuse parenchymal lung disease as first clinical manifestation of GATA-2 deficiency in childhood. BMC Pulm Med 2015; 15: 8.

Copyright @ERS 2017

From the authors:

Over the past few years, genetics has significantly improved the understanding of interstitial lung diseases (ILD). For example, in idiopathic pulmonary fibrosis, telomerase complex mutations have been shown to drive a specific phenotype including haematological, liver and cutaneous abnormalities [1]. Recently, GATA2 deficiency has been associated with a broad phenotype including haematological, vascular, infectious and pulmonary diseases [2-4]. GATA2 is a zinc finger transcription factor essential for differentiation of immature haematopoietic cells. Among many other functions, GATA2 regulates the phagocytosis of alveolar macrophages. Therefore, alveolar macrophage dysfunction is thought to be the pathophysiological basis for the occurrence of pulmonary alveolar proteinosis, the main lung condition in GATA2 deficiency [4]. However, pulmonary alveolar proteinosis associated with GATA2 deficiency does not share the same clinical, biological or radiological features as autoimmune pulmonary alveolar proteinosis. In all cases of GATA2 deficiency-associated pulmonary alveolar proteinosis reported in the literature, granulocyte-macrophage colony-stimulating factor (GM-CSF) antibodies were absent $[2,4]$. Moreover, other pulmonary diseases such as fibrosis or pulmonary hypertension (PH) have been reported to occur in GATA2 deficiency [4], suggesting that the precise pathophysiological mechanisms are not fully understood.

S. Sanges and colleagues report the case of a 41-year-old patient with GATA2 deficiency, and histologically proven pulmonary alveolar proteinosis and pulmonary fibrosis, complicated with haemodynamically proven PH. As PH was considered secondary to the lung disease (World Health Organization group 3), specific pulmonary arterial hypertension therapies were not introduced [5]. As highlighted by S. Sanges and colleagues, physicians should be aware of the possible occurrence of PH in patients with GATA2 deficiency, as well as in every patient presenting with chronic fibrosing ILD.

What is striking in this adult case, as in our previous report [6], is the computed tomography (CT) appearance of ILD. Indeed, reticulations (i.e. pulmonary fibrosis) are the predominant pattern and there is no "crazy paving". In both cases, chest CT was not suggestive of pulmonary alveolar proteinosis and the diagnosis was finally made by surgical lung biopsy.

Another recent case from Bordeaux Academic Hospital (Bordeaux, France) illustrates the same points (X. Demant and co-workers, unpublished observations): a 21-year-old patient with chest CT scan showing fibrosing ILD (subpleural and perihilar reticulations associated with some areas of ground-glass opacity without typical crazy paving) and a diagnosis of pulmonary alveolar proteinosis made on surgical lung biopsy. Associated severe monocytopenia and lymphopenia led to the diagnosis of GATA2 deficiency.

$@$ ERSpublications

Pulmonary fibrosis may be more prevalent in adults with GATA2 deficiency due to a longer duration of the disease http://ow.ly/WHnu30aCZ4S

Cite this article as: Jouneau S, Ballerie A, Kerjouan M, et al. Haemodynamically proven pulmonary hypertension in a patient with GATA2 deficiency-associated pulmonary alveolar proteinosis and fibrosis. Eur Respir J 2017; 49: 1700407 [https://doi.org/10.1183/13993003.00407-2017]. 
Traditionally, crazy paving, defined as the superimposition of reticular lines on ground-glass opacity, is considered the cardinal CT sign of pulmonary alveolar proteinosis, leading to bronchoalveolar lavage to confirm the diagnosis. Although crazy paving has been described in GATA2 deficiency-associated pulmonary alveolar proteinosis [2, 4], it appears less frequent and less typical than in autoimmune pulmonary alveolar proteinosis. Moreover, pulmonary complications such as end-stage fibrosis or $\mathrm{PH}$ are not uncommon in GATA2 deficiency, while they are quite rare in autoimmune pulmonary alveolar proteinosis.

Therefore, the spectrum of pulmonary CT findings in GATA2 deficiency appears to be more heterogeneous than in autoimmune pulmonary alveolar proteinosis, ranging from "pure" crazy paving to severe lung fibrosis, potentially complicated by PH. A fibrosis-predominant phenotype may be more prevalent in adults due to a longer duration of pulmonary alveolar proteinosis, as illustrated by the three cases presented herein. Furthermore, pulmonary fibrosis as final evolution of pulmonary alveolar proteinosis has been described, especially in old case reports [7, 8]. However, because most patients with GATA2 deficiency-associated pulmonary alveolar proteinosis exhibit a severe phenotype including myelodysplastic syndrome as well as immunodeficiency and recurrent infections [4], confounding factors might also account for the association between pulmonary alveolar proteinosis and fibrosis. The occurrence of $\mathrm{PH}$ seems to be less frequent than that of pulmonary alveolar proteinosis and fibrosis but might be underdiagnosed. Further research is necessary to investigate whether GATA2 deficiency might be an independent risk factor for PH.

Pulmonologists should be aware of the severe and heterogeneous clinical spectrum associated with GATA2 deficiency. As allogenic haematopoietic stem cell transplantation seems to be the only efficient treatment to improve the respiratory condition, it is crucial that patients with pulmonary alveolar proteinosis without GM-CSF antibodies are screened for GATA2 deficiency. Likewise, patients exhibiting fibrosing ILD, with or without $\mathrm{PH}$, associated with haematological abnormalities need to benefit from early genetic diagnosis for appropriate therapeutic management as well as family screening.

Stéphane Jouneau ${ }^{1,2}$, Alice Ballerie $\oplus^{2,3}$, Mallorie Kerjouan ${ }^{1}$, Xavier Demant ${ }^{4}$, Elodie Blanchard ${ }^{4}$ and Mathieu Lederlin ${ }^{5,6}$

${ }^{1}$ Dept of Respiratory Medicine, Pontchaillou Hospital, Rennes 1 University, Rennes, France. ${ }^{2}$ IRSET UMR1085, Rennes 1 University, Rennes, France. ${ }^{3}$ Dept of Internal Medicine, Pontchaillou Hospital, Rennes 1 University, Rennes, France. ${ }^{4}$ Dept of Respiratory Medicine, Haut-Lévèque Hospital, Bordeaux University, Pessac, France. ${ }^{5}$ Dept of Radiology, Pontchaillou Hospital, Rennes 1 University, Rennes, France. ${ }^{6}$ LTSI, INSERM U1099, Rennes 1 University, Rennes, France.

Correspondence: Stéphane Jouneau, Dept of Respiratory Medicine, Pontchaillou Hospital, Rennes 1 University, 2 rue Henri Le Guilloux, 35033 Rennes Cedex 9, France. E-mail: Stephane.jouneau@chu-rennes.fr

Received: Feb 252017 | Accepted after revision: March 052017

Conflict of interest: None declared.

\section{References}

1 Borie R, Tabeze L, Thabut G, et al. Prevalence and characteristics of TERT and TERC mutations in suspected genetic pulmonary fibrosis. Eur Respir J 2016; 48: 1721-1731.

2 Griese M, Zarbock R, Costabel U, et al. GATA2 deficiency in children and adults with severe pulmonary alveolar proteinosis and hematologic disorders. BMC Pulm Med 2015; 15: 87.

3 Hsu AP, Sampaio EP, Khan J, et al. Mutations in GATA2 are associated with the autosomal dominant and sporadic monocytopenia and mycobacterial infection (MonoMAC) syndrome. Blood 2011; 118: 2653-2655.

4 Spinner MA, Sanchez LA, Hsu AP, et al. GATA2 deficiency: a protean disorder of hematopoiesis, lymphatics, and immunity. Blood 2014; 123: 809-821.

5 Galie N, Humbert M, Vachiery JL, et al. 2015 ESC/ERS Guidelines for the diagnosis and treatment of pulmonary hypertension. Eur Respir J 2015; 46: 903-975.

6 Ballerie A, Nimubona S, Meunier C, et al. Association of pulmonary alveolar proteinosis and fibrosis: patient with GATA2 deficiency. Eur Respir J 2016; 48: 1510-1514.

7 Hudson AR, Halprin GM, Miller JA, et al. Pulmonary interstitial fibrosis following alveolar proteinosis. Chest 1974; 65: 700-702.

8 Clague HW, Wallace AC, Morgan WK. Pulmonary interstitial fibrosis associated with alveolar proteinosis. Thorax 1983; 38: 865-866. 